\title{
ACTUALIZACIÓN DE LOS REGISTROS AFIDOLÓGICOS (HOMOPTERA: APHIDOIDEA) DE LA PROVINCIA DE SANTA FE, ARGENTINA ${ }^{1}$
}

\author{
Ortego, J. ${ }^{2}$, Paravano, A. S. ${ }^{3}$
}

\& IMWINKELRIED, J. M. ${ }^{4}$

\begin{abstract}
RESUMEN
Un relevamiento de pulgones se realizó en la ciudad de Esperanza, Santa Fe, por dos metodologías diferentes: inspección de plantas hospederas y capturas con trampas amarillas de agua. Se identificaron 26 especies diferentes de las que 10 resultaron nuevas para Santa Fe y una de ellas representa un nuevo registro para el continente americano.

Palabras claves: áfidos, Santa Fe, trampas amarillas de agua, Melanaphis bambusae (Fullaway, 1910)
\end{abstract}

\section{SUMMARY}

\section{Actualization of the aphidologyc records (Hemiptera: Aphidoidea) of Santa Fe province, in Argentina.}

A survey on aphid species was made in Esperanza city, province of Santa $\mathrm{Fe}$, using two different methodologies: host plants inspections and catches in Yellow Water Traps. It has been identified 26 different species. Ten species are new records for Santa Fe and one is a new one for the American continent.

Key words: aphids, Santa Fe, yellow water traps; Melanaphis bambusae (Fullaway, 1910).

1.- Trabajo realizado como parte del "Curso-Taller sobre Identificación y Bioecología de Homópteros que Afectan a los Principales Cultivos de la Región Central de la Provincia de Santa Fe", Facultad de Ciencias Agrarias (UNL), Esperanza, Santa Fe.

2.- INTA AER Malargüe; S. Torres 862, 5613 Malargüe, Mendoza, Argentina.

3.- Cátedra den Zoología Agrícola de la Facultad de Ciencias Agrarias de la Universidad del Litoral. R. P. Kreder 2805, 3080 Esperanza, Santa Fe, Argentina.

4.- INTA EEA Rafaela; CC N 22; 2300 Rafaela, Santa Fe, Argentina.

Manuscrito recibido el 5 de abril de 2000 y aceptado para su publicación el 10 de noviembre de 2001. 